\title{
A Modified Context based Image Interpolation Algorithm for Digital Images
}

\author{
Sandeepa K. S. \\ Research Scholar \\ Department of Electronics \\ Kuvempu University, Karntaka, \\ India
}

\author{
B. N. Jagadale \\ Assistance Professor \\ Department of Electronics \\ Kuvempu University, Karntaka, \\ India
}

\author{
J. S. Bhat \\ Professor \\ Department of Physics \\ Karnataka University, Karntaka, \\ India
}

\begin{abstract}
A modified context based interpolation algorithm, for digital images, is presented. In the proposed algorithm, the unknown pixel value is exploited based upon the characteristic of a neighboring pixel by considering its edge. The edge is obtained by taking differences of two slopes calculated from neighboring pixels, which are in orthogonal direction. The algorithm uses fourth ordered prediction based approach when interpolating new pixel value by giving suitable weights to the neighboring pixels. This method gives better results as compared to some of existing interpolation methods. Comparison has done using Peak Signal to Noise Ratio (PSNR) and Correlation Coefficient method.
\end{abstract}

\section{General Terms}

Image interpolation is widely used in various applications such as remote sensing, computer graphics, rendering, editing medical images and surveillances.

\section{Keywords}

Prediction coefficient, interpolation, low resolution image, neighboring pixels, slope identification, weight estimator.

\section{INTRODUCTION}

Image interpolation deals with assigning valid pixel value in place of a missing one, while creating high resolution image from a low resolution image without losing the visual content of the picture.

Some of the classical interpolation techniques such as bilinear, bicubic, spline [1], [2], [3] are used in many real time applications. However, these methods are based on some spatial invariant model and fail to capture the edge information of an image. Various interpolation algorithms have been developed so far: Li and Orchard [4] suggested the edge directed interpolation algorithm, in which the missing pixels are interpolated based on the estimated covariance of the High Resolution (HR) image from the covariance of Low Resolution (LR) image (NEDI). Alternatively, Zhang and Wu proposed an Image Interpolation algorithm based on the Adaptive 2-D Autoregressive Modeling and Soft-Decision Estimation (SAI) [5]. Jakhetiya and Tiwari proposed a single pass interpolation algorithm (SPIA) [6] based on least square estimation. Tai-Wai Chan, Oscar C. Au, et all proposed a novel content-adaptive interpolation algorithm (CAI) [7]. Jakhetiya et all proposed a computational efficient context based switched image interpolation for natural image [8]. In which edge image and smooth image are interpolated separately by the creations of bins.

In the proposed algorithm, edge is considered as the key issue and the method, assigns adaptive weight to a pixel, based on the correlation of the neighboring pixels.
The paper is organized as follows: In section 2 the particulars of the algorithm is discussed. The simulation result are given and discussed in section 3 and Conclusions, future scope are presented in section 4 .

\section{PROPOSED ALGORITHM}

This work modifies the context based interpolation algorithm by considering all valid neighboring pixel values. Here edge and smoothness is interpreted in a simple concept. First detect edge direction across the pixel that to be interpolated, and then give more adaptive weight to the direction where less edge is found and vice versa. In this method, interpolating missing pixels of high resolution image is done in two steps. In the first step we consider odd-odd pixel position to interpolate entire image as shown in figure 1 and assigning adaptive weight to particular pixel depends on block considered, as shown in figure 2. In second step, for interpolation of remaining pixels a different approach is adapted as shown in figure 3 . With the help of original and new interpolated value from first step, adaptive weight of each of the remaining pixel decided as shown in the figure 4 .

\subsection{The first step}

\subsubsection{Slope identification}

In the first step, the identification of the edge direction with the help of neighboring pixels shown in figure 1 , in terms of slope $S_{D 1}$, as given by.

$$
S_{D 1}=\left(d_{O 1}-d_{O 2}\right),
$$

where

$$
\begin{aligned}
& \left.d_{O 1}=\mid K-G\right\rfloor+|G-C|+|C-B|+|B-F|+|F-J| \\
& d_{O 2}=|I-E|+|E-A|+|A-D|+|D-H|+|H-L|
\end{aligned}
$$

The value of $S_{D 1}$ gives the edge direction, When $S_{D 1}$ is positive, that edge is along $d_{O 1}$, otherwise edge is along $d_{O 2}$ . If $S_{D 1}$ is zero, that it indicates current pixel has same value as that of neighboring pixels.

\subsubsection{Interpolating missing pixels on weight basis}

The unknown pixel value is obtained using fourth order prediction based approach. The method is described as follows:

1. Indentifying the unknown pixel and its edge direction based on slope value $S_{D 1}$. 


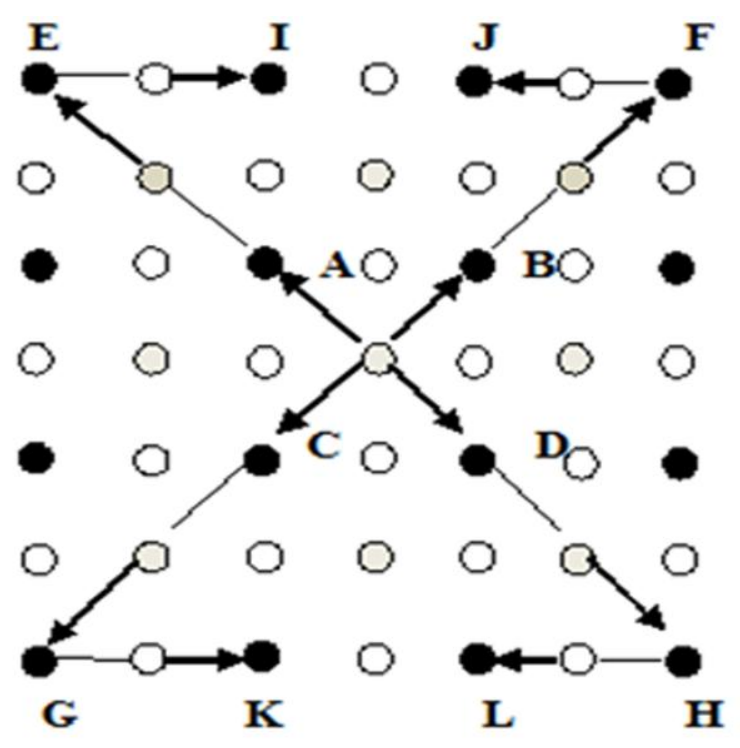

Fig.1: Neighboring pixels (context) used for identifying edge and interpolating new pixels.

2. Depending on the edge direction assign suitable adaptive weight to all neighboring pixels. It is achieved by weight estimator as shown in figure 2 , in terms of $S_{w 1}$, which is computed as

If $S_{D 1}$ is positive

$$
S_{w 1}=A_{1}-B_{1}
$$

where,

$$
\begin{gathered}
A_{1}=|E-I|+|E-A|+|I-A| \\
B_{1}=|D-L|+|L-H|+|H-D|
\end{gathered}
$$

If $S_{D 1}$ is negative

$$
S_{w 1}=C_{1}-D_{1}
$$

where,

$$
\begin{aligned}
C_{1} & =|C-G|+|G-K|+|K-C| \\
D_{1} & =|J-B|+|B-F|+|F-J|
\end{aligned}
$$

If $S_{D 1}$ is zero

\section{Unknown pixel has same value like its neighboring pixels}

3. The unknown pixels are predicted with the help of prediction coefficients $\left(\alpha_{1}, \alpha_{2}, \alpha_{3}, \alpha_{4}\right)$, which depend on the positive and negative value of the weight estimator $S_{w 1}$ (Table1).

4. Interpolate all missing pixels using the equation

$$
x(n)=\alpha_{1} A+\alpha_{2} B+\alpha_{3} C+\alpha_{4} D
$$

Half of the high resolution (HR) pixels are obtained in the first step of interpolation process, while remaining half of the HR missing pixels will be interpolated in second step.

\subsection{The second step}

\subsubsection{Slope identification}

The interpolation process in this step is essentially similar to first step. The only difference is that the proposed algorithm will interpolate the missing HR pixels with the help of four neighboring pixels as shown in Figure 3, in terms of $S_{D 2}$ as shown in equation (5), which are either known or estimated in the first step:

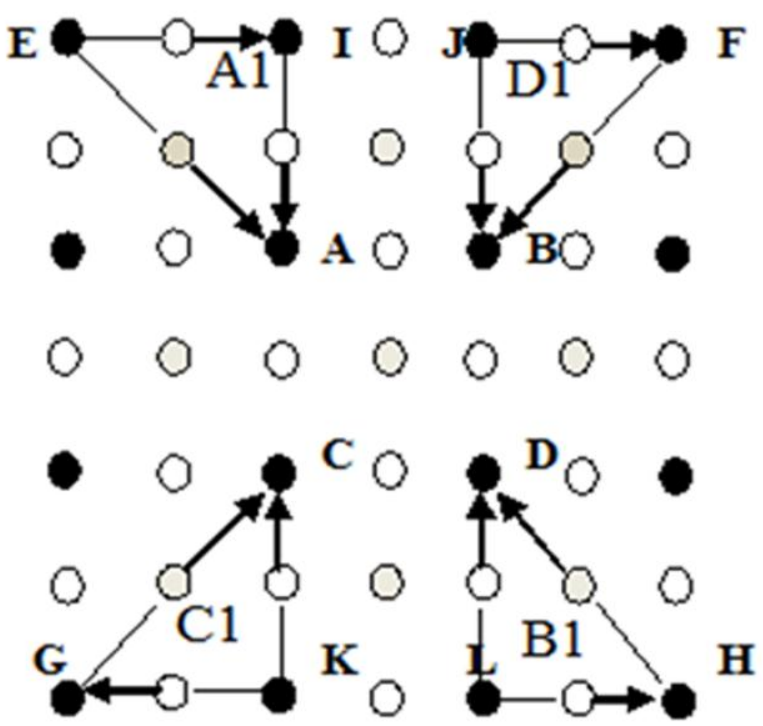

Fig. 2: Adaptive weight assigning based on the edge along the diagonal direction.

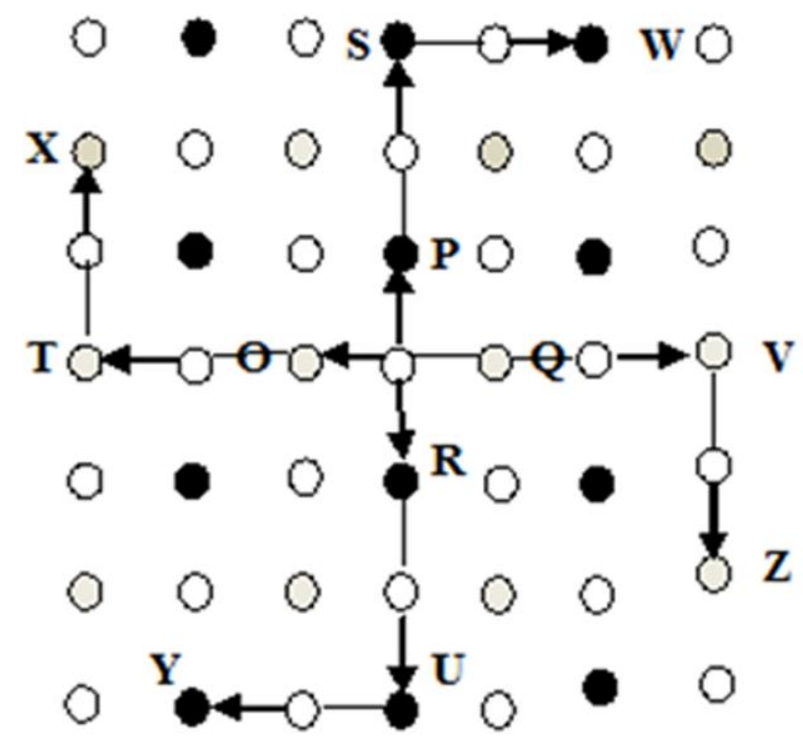

Fig.3: Six connected neighboring pixels used for interpolation process.

$$
S_{D 2}=d_{h}-d_{v}
$$

where

$$
\begin{aligned}
& d_{h}=|Y-U|+|U-R|+|R-P|+|P-S|+|S-W| \\
& d_{V}=|Z-V|+|V-Q|+|Q-O|+|O-T|+|T-X|
\end{aligned}
$$

2.2.2 Interpolating missing pixels on weight basis The adaptive weight is assigned to all neighboring pixels by using weight estimator as shown in figure 4 depending on the direction obtained by $S_{D 2}$ :

If $S_{D 2}$ is positive, then

$$
S_{w 1}=C 1-D 1
$$

where, $\quad C 1=|C-E|+|E-O|+|C-O|$

$$
D 1=|Q-H|+|H-J|+|J-Q|
$$




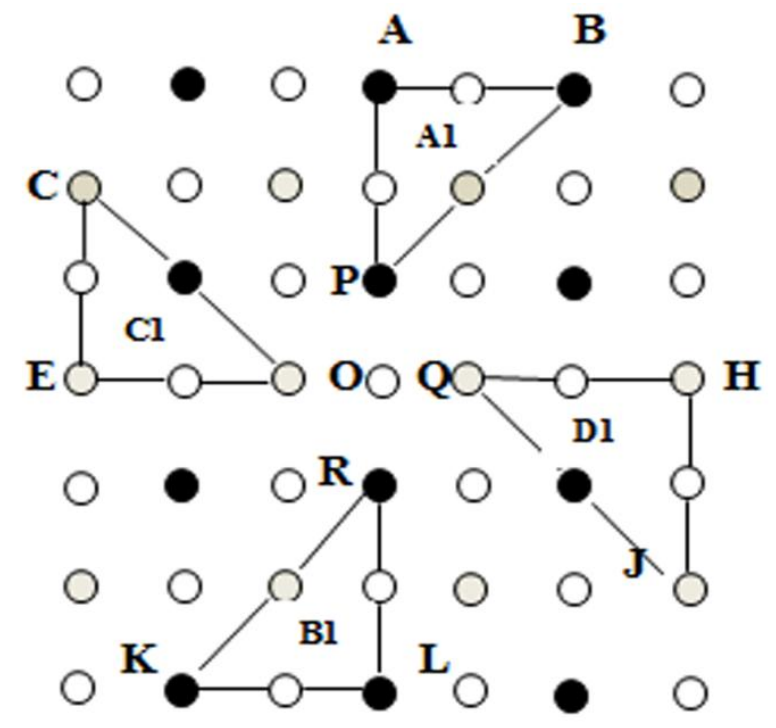

Fig.4: Assigning of adaptive weight to neighboring pixels.

If $S_{D 2}$ is negative, then

$$
S_{w 1}=A 1-B 1
$$

where,

$$
\begin{gathered}
A 1=|A-B|+|B-P|+|P-A| \\
B 1=|R-L|+|K-L|+|L-R|
\end{gathered}
$$

If $S_{D 2}$ is zero, then

Unknown pixel get its neighboring pixels value.

All missing pixels are interpolated using equation (8), with the help of prediction coefficients given in Table 1.

$$
x(n)=\alpha_{1} P+\alpha_{2} Q+\alpha_{3} R+\alpha_{4} O
$$

The corner four pixels along rows and columns are

\begin{tabular}{|c|c|c|c|c|}
\hline \multicolumn{5}{|c|}{ Edge along diagonal direction $d_{01}$} \\
\hline$S_{w 1}$ & $\alpha_{1}$ & $\alpha_{2}$ & $\alpha_{3}$ & $\alpha_{4}$ \\
\hline Positive & 0.2588 & 0.2693 & 0.2374 & 0.2377 \\
\hline negative & 0.2693 & 0.2588 & 0.2374 & 0.2377 \\
\hline If equal & 0.2674 & 0.2674 & 0.2374 & 0.2199 \\
\hline \multicolumn{5}{|c|}{ Edge along diagonal direction $d_{O 2}$} \\
\hline$S_{w 1}$ & $\alpha_{1}$ & $\alpha_{2}$ & $\alpha_{3}$ & $\alpha_{4}$ \\
\hline Positive & 0.2374 & 0.2693 & 0.2588 & 0.2377 \\
\hline negative & 0.2374 & 0.2588 & 0.2693 & 0.2377 \\
\hline If equal & 0.2374 & 0.2674 & 0.2674 & 0.2199 \\
\hline \multicolumn{5}{|c|}{ Edge along horizontal direction $d_{h}$} \\
\hline$S_{w 1}$ & $\alpha_{1}$ & $\alpha_{2}$ & $\alpha_{3}$ & $\alpha_{4}$ \\
\hline Positive & 0.2374 & 0.2693 & 0.2377 & 0.2588 \\
\hline negative & 0.2374 & 0.2588 & 0.2377 & 0.2693 \\
\hline If equal & 0.2374 & 0.2674 & 0.2199 & 0.2674 \\
\hline \multicolumn{5}{|c|}{ Edge along vertical direction $d_{v}$} \\
\hline$S_{w 1}$ & $\alpha_{1}$ & $\alpha_{2}$ & $\alpha_{3}$ & $\alpha_{4}$ \\
\hline Positive & 0.2588 & 0.2374 & 0.2693 & 0.2377 \\
\hline negative & 0.2693 & 0.2374 & 0.2588 & 0.2377 \\
\hline If equal & 0.2674 & 0.2374 & 0.2674 & 0.2199 \\
\hline
\end{tabular}
interpolated using bilinear interpolation method. High resolution output image is obtained by completing both steps.

Table 1: Prediction coefficient for $\left(\alpha_{1}, \alpha_{2}, \alpha_{3}\right.$ and $\left.\alpha_{4}\right)$

\section{SIMULATION RESULT}

The proposed modified context based interpolation algorithm is implemented using gray scale test images [9]. The comparisons are made based on PSNR and Correlation coefficients with some standard interpolation techniques and results are tabulated in Table $2 \& 3$ respectively. The average PSNR and Correlation Coefficient value of all the eight images calculated and tabulated.

The proposed algorithm gives better PSNR and correlation coefficient values than those obtained by using Bicubic, Bilinear, CAI and CBID [10], respectively. The images are interpolated from Low resolution 256X256 to High resolution 512X512. The input low resolution image and high resolution output images are shown in the figure 5 and 6 .

Table 2: PSNR Comparison of Images Interpolated by Different Methods

\begin{tabular}{|l|l|l|l|l|l|}
\hline $\begin{array}{l}\text { image } \\
\boldsymbol{s}\end{array}$ & bicubic & bilinear & CAI & CBID & $\begin{array}{l}\text { propos } \\
\text { ed }\end{array}$ \\
\hline 1 & 31.994 & 32.768 & 32.689 & 32.827 & $\mathbf{3 2 . 8 5 3}$ \\
\hline 2 & 28.487 & 28.193 & 28.188 & 28.503 & $\mathbf{2 9 . 7 4 9}$ \\
\hline 3 & 30.796 & 30.088 & 32.121 & 31.932 & $\mathbf{3 2 . 6 3 2}$ \\
\hline 4 & 21.727 & 21.875 & 21.287 & 21.881 & $\mathbf{2 2 . 0 5 4}$ \\
\hline 5 & 22.523 & 22.871 & 22.279 & 22.861 & $\mathbf{2 2 . 9 6 7}$ \\
\hline 6 & 22.723 & 22.616 & 22.238 & 22.614 & $\mathbf{2 3 . 2 3 4}$ \\
\hline 7 & 28.521 & 28.190 & 28.851 & 28.948 & $\mathbf{3 0 . 2 3 8}$ \\
\hline 8 & 29.263 & 28.672 & 28.716 & 29.269 & $\mathbf{2 9 . 7 9 9}$ \\
\hline aver & 27.004 & 26.909 & 26.921 & 27.354 & $\mathbf{2 7 . 9 4 0}$ \\
\hline
\end{tabular}

Table 3: Correlation Coefficient of the HR Images Compared with Different Interpolation Algorithm

\begin{tabular}{|l|l|l|l|l|l|}
\hline images & bicubic & bilinear & CAI & CBID & proposed \\
\hline 1 & 0.9715 & 0.9758 & 0.9754 & 0.9762 & $\mathbf{0 . 9 7 6 2}$ \\
\hline 2 & 0.9737 & 0.9722 & 0.9718 & 0.9718 & $\mathbf{0 . 9 8 0 7}$ \\
\hline 3 & 0.9907 & 0.9890 & 0.9913 & 0.9928 & $\mathbf{0 . 9 9 3 9}$ \\
\hline 4 & 0.8862 & 0.8878 & 0.8747 & 0.8880 & $\mathbf{0 . 8 9 2 6}$ \\
\hline 5 & 0.9541 & 0.9575 & 0.9519 & 0.9574 & $\mathbf{0 . 9 5 8 4}$ \\
\hline 6 & 0.8995 & 0.8950 & 0.8872 & 0.8950 & $\mathbf{0 . 9 0 9 7}$ \\
\hline 7 & 0.9791 & 0.9805 & 0.9804 & 0.9809 & $\mathbf{0 . 9 8 8 8}$ \\
\hline 8 & 0.9905 & 0.9891 & 0.9892 & 0.9905 & $\mathbf{0 . 9 9 1 6}$ \\
\hline aver & 0.9556 & 0.9564 & 0.9527 & 0.9565 & $\mathbf{0 . 9 6 1 4}$ \\
\hline
\end{tabular}

a
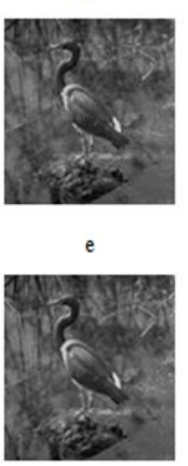

b

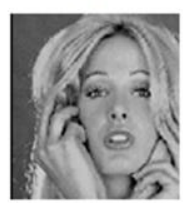

f

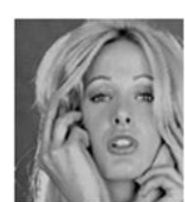

c

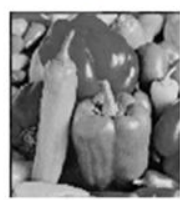

$\mathrm{g}$

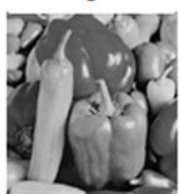

d

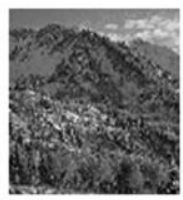

$\mathrm{h}$

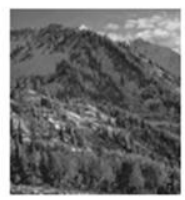

Fig.5: (a-d): Original Low Resolution image, (e-h): High Resolution 512x512 output image 

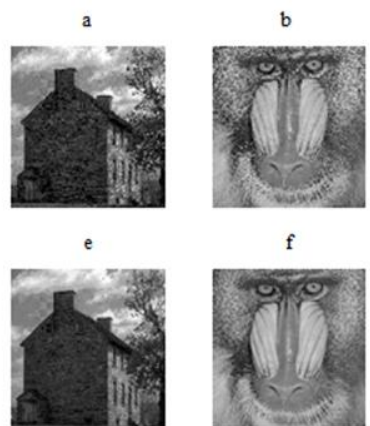

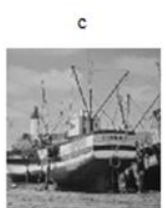

g

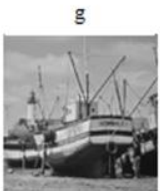

Fig.6: (a-d): Original Low Resolution image, (e-h): High Resolution $512 \times 512$ output image

\section{CONCLUSION AND FUTURE SCOPE}

In this paper, a modified context based interpolation method is presented. The proposed algorithm is a simple and efficient method to enlarge images. The proposed algorithm follows the procedure of classifying unknown pixels as in CBI, but it avoids creation of bin. The method operates in two steps, first considering its odd-odd pixels and assigning adaptive weight to the particular pixels depending on the weight estimator value. In the second steps operates on remaining pixels with the same procedure. The weight estimator gives the direction, where exactly weight has to apply in the consider block. The method is also able to reconstruct sharp edge accurately in enlarged image and shows significant improvement in PSNR values compared to some of the existing interpolation techniques with a marginal increment of computation time.

A good and efficient methodology for image interpolation, further improvement can be in corner image pixel formation and reduction in the steps for algorithm implementation is possible to minimize the computation time. The proposed algorithm takes two steps for interpolating total image. If it implemented in single step, algorithm can be more optimized.

\section{ACKNOWLEDGMENTS}

The research work is supported by UGC -MRP, New Delhi, India.

\section{REFERENCES}

[1] E. Maeland, "On the comparison of interpolation methods" IEEE Transactions on Medical Imaging, vol. 7 , no. 3, September, 1988.

[2] Hou, and H. Andrews, "Cubic splines for image interpolation and digital filtering" IEEE Transactions on Acoustics, Speech, and Signal Processing, vol. 26, no. 6, pp. 508-517,1978.

[3] R. Keys, "Cubic convolution interpolation for digital image processing" IEEE Transactions on Acoustics, Speech, and Signal Processing, vol. 29, no. 6, pp. 11531160,1981

[4] Xin Li and Michael T. Orchard "New Edge-Directed Interpolation, in IEEE Transaction On Image Processing, Vol. 10, No. 10, October 2001.

[5] Xiangjun Zhang and Xiaolin Wu "Image Interpolation by Adaptive 2- D Autoregressive Modeling and SoftDecision Estimation," in IEEE Transaction On Image Processing, Vol. 17, No. 6, June 2008.

[6] Vinit Jakhetiya and Anil K. Tiwari, "Image interpolation by adaptive 2 -D autoregressive modeling," in International Conference on Digital Image Processing, 2010 .

[7] Tai-Wai Chan; Au, O.C.; Tak-Song Chong; Wing-San Chau; "A novel content-adaptive interpolation," in IEEE International Symposium on Circuits and Systems, 2005. , vol., no., pp. 6260- 6263 Vol. 6.

[8] Jakhetiya, V.; Jaiswal, S.P.; Tiwari, A.K. "A computationally efficient context based switched image interpolation algorithm for natural images, in I2MTC, 2011 IEEE , vol., no., pp.1-4, 10-12 May 2011.

[9] Dataset of Standard 512x512 Grayscale Test Images. Universidad de http://decsai.ugr.es/cvg/CG/base.htm

Granada:

[10] Sunil Prasad Jaiswal, yVinit Jakhetiya, _Ayush Kumar, zAnil Kumar Tiwari "A Low Complex Context Adaptive Image Interpolation Algorithm for Real-Time Applications" in IEEE international conference 2012. 